\title{
Une artiste sans galerie
}

Un entretien avec Sanja Iveković

\section{Sanja Iveković}

\section{(2) OpenEdition \\ Journals}

Édition électronique

URL : http://journals.openedition.org/conflits/18609

DOI : 10.4000/conflits.18609

ISSN : $1777-5345$

Éditeur :

CCLS - Centre d'études sur les conflits lilberté et sécurité, L'Harmattan

Édition imprimée

Date de publication : 30 décembre 2012

Pagination : 159-172

ISBN : 978-2-343-00589-8

ISSN : 1157-996X

Référence électronique

Sanja Iveković, «Une artiste sans galerie », Cultures \& Conflits [En ligne], 88 | hiver 2012, mis en ligne le 29 janvier 2013, consulté le 30 mars 2021. URL : http://journals.openedition.org/conflits/18609 ; DOI : https://doi.org/10.4000/conflits.18609 
REGARDS SUR L'ENTRE-DEUX 



\section{Une artiste sans galerie Entretien avec Sanja Iveković}

Entretien réalisé par Rada Iveković, philosophe (Paris), traduit par Goran Feji.

Le travail de Sanja Iveković est marqué par le discours critique envers les politiques de l'image et du corps. L'analyse des constructions identitaires par les médias tout comme l'engagement politique, la solidarité et l'activisme sont des thèmes qui traversent ses stratégies artistiques. Elle travaille avec des performances, des vidéos, des installations et des actions dans le domaine public depuis les années 1970. Son travail depuis les années 1990 aborde l'effondrement des régimes socialistes et les conséquences du triomphe du capitalisme et de l'économie de marché sur les conditions de vie, notamment des femmes. Parmi ses expositions récentes : «Waiting for the Revolution ", MUDAM, Luxembourg, "Sweet Violence », MoMA, New York (2011), dokumenta 13 (2012) ; " Practice Makes a Master », Museum Sztuki, Lodz ; «Urgent Matters », Bak, Van Abbemusem, (2009) ; "General Alert », Kölnischer Kunstverein ; Fundacio Antoni Tapies, Gothenburg Konsthall; Dokumenta 12, Kassel (2007) ; "Public Cuts », Galerija P74, Ljubljana (2006); «Open Systems: Rethinking Art c. $1970 »$; Tate Modern, London ; «Die Regierung », Secession (2005); «Women’s House », Palazzo Ferreri, Gênes (2004) ; documenta 11, Kassel ; «Personal Cuts » NGBK (2002) ; Galerie im Taxispalais, Innsbruck (2001) ; «After the Wall », Moderna Muset, (1999/2001), Manifesta 2, Luxembourg (1998).

Rada Iveković (R. I.) : Lors de ta résidence au MAC/VAL ${ }^{1}$ en 2012, tu t'es présentée comme née à Zagreb, Yougoslavie, résidant à Zagreb, Croatie. $T u$ as résisté avec vébémence à la récupération par l'État (en l'occurrence croate) de ton travail : tu n'as pas soubaité faire partie du programme "La

1. http://www.macval.fr/francais/residences-commandes/article/sanja-ivekovic; MAC/VAL, Musée d'art contemporain du Val de Marne, http://www.macval.fr/. 
Croatie, la voici! " pour ta performance, et tu ne t'es pas présentée à ton propre vernissage pour ton installation "Visages du langage " 2, qui montrait des écrans avec des animaux, autant d'appellations injurieuses des femmes; ces animaux se transformaient en visages de femmes battues. Tu as présenté une performance intitulée "Pourquoi un(e) artiste ne peut représenter un Étatnation " ${ }^{3}$, à propos de laquelle tu t'es distanciée du programme officiel de la Croatie en France. On peut dire que dans l'ensemble, ton travail a toujours invité à la réflexion politique. Voici la question : depuis quand refuses-tu d'être identifiée à la nation et à l'État-nation?

Sanja Iveković (S. I.) : Je dis toujours que je suis née à Zagreb, en Yougoslavie et que je vis et travaille à Zagreb, en Croatie. J'ai vécu 45 années en Yougoslavie et je ne veux pas mentir. La création de l'État croate en 1991 (la Croatie a été admise à l'ONU en 1992) est allée de pair avec le bouillonnement d'un discours nationaliste et une re-patriarcalisation de la société, tandis que le canon préféré de l'art devenait celui qui consacrait « la renaissance de la nation ». J'ai été élevée dans un esprit cosmopolite et je ne voulais pas m'identifier avec une telle politique. J'ai toujours été critique envers le gouvernement, qu'il soit socialiste ou nationaliste et cela m'a causé bien des ennuis. Avant la guerre, j'ai travaillé en tant que graphiste dans une maison d'édition. Quand la guerre a commencé, j'étais en voyage d'étude au Canada et je ne suis pas rentrée à temps en Croatie pour être « recensée » comme étant « du bon côté ». J'ai donc été virée. Plus tard, ma demande de statut d'artiste indépendant a été rejetée. C'était plutôt choquant parce que j'étais déjà, à l'époque, une artiste reconnue. On m'a dit que mon séjour à l'étranger prouvait que je n'étais pas une bonne patriote. J'ai compris que le nouveau gouvernement me traitait de dissidente. Cependant, je ne voulais pas me résigner à ce statut, à l'inactivité, au retrait et à la non-participation. D'autre part, comme le discours nationaliste était omniprésent pendant ces années 1990, je ne voulais pas, non plus, exposer dans les galeries d'État. Dans cette situation, j'ai changé d'approche. Mon travail de cette époque reste encore apprécié aujourd'hui. Je fais référence à l'idée de ma réalisation «Le gène XX », à la conception du Magazine indépendant «ARKZIN », lancé d'abord comme pamphlet antiguerre et qui est devenu ensuite une importante " poche de résistance » à la frénésie militariste et nationaliste. Dans cette œuvre ("Gène XX ») j'aborde un thème qui était en train de devenir presque tabou à l'époque : celui de notre héritage antifasciste. Comme tout ce qui datait de l'époque socialiste, cet héri-

2. «Visages du langage », installation à partir du 19 octobre 2012, MAC/VAL.

3. Sanja Iveković, "Pourquoi un(e) artiste ne peut représenter un État-nation », avec la participation de Rada Iveković (philosophe) et d'Isabelle Voizeux (comédienne), performance en partenariat avec le Festival d'Automne de Paris, le 21 octobre 2012 à 17h30. L'installation ainsi que la performance ont été soutenues par la curatrice Valérie Labayle du MAC/VAL (que nous remercions ici) qui a également mené les entretiens du "Dossier de presse Automne 2012 ». Voir sur «Le beau vice » le blog d'Elisabeth Lebovici : «Pourquoi un(e) artiste ne peut représenter un État-nation »: http://le-beau-vice.blogspot.fr/2012/10/pourquoi-une-artiste-ne-peut.html 
tage devenait l'objet d'une véritable amnésie collective. C'est pendant ces mêmes années que j’ai commencé à collaborer avec les ONG féministes dont la politique s'opposait clairement aux visées nationalistes. Je suis fière aujourd'hui d'avoir personnellement contribué à la création de certaines de ces organisations.
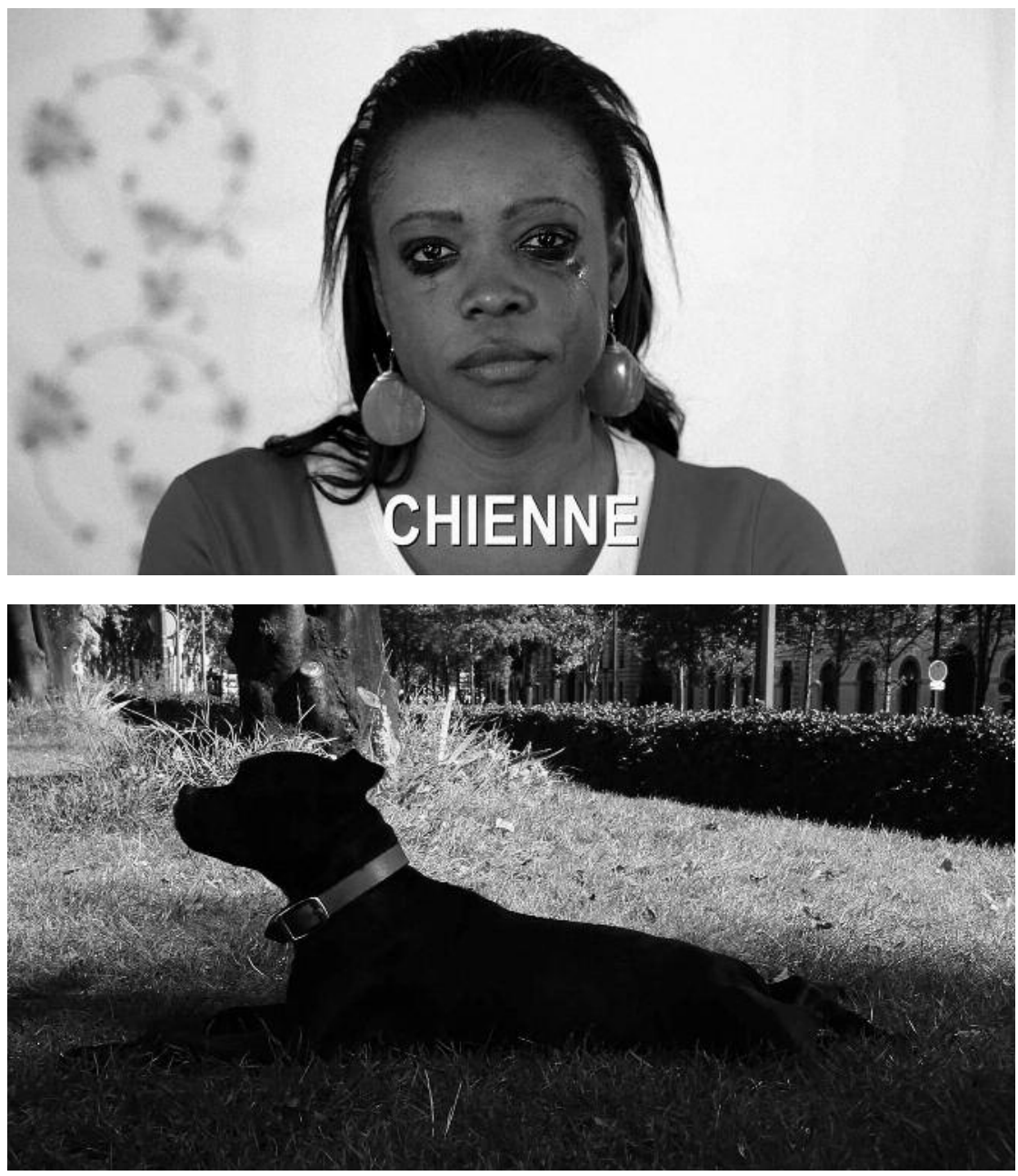

"Chienne »- Ces photographies font partie du travail "Visages du Langage », installation de Sanja Iveković, octobre 2012, MAC/VAL (C) Marko Ercegović 
R. I. : Lors de la préparation des dossiers de presse et de ta présentation en France, une certaine ignorance a été déployée par des instances organisatrices

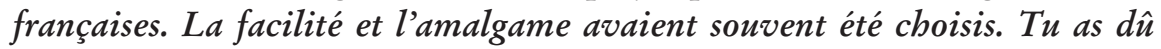
intervenir pour rectifier des clichés selon lesquels la Yougoslavie aurait été en proie au réalisme socialiste ou aurait fait partie du bloc soviétique. Selon ces clichés, Sanja Iveković aurait combattu en tant qu'artiste le réalisme socialiste, alors qu'on passait sous silence ta critique de la société capitaliste de consommation ou des nationalismes, non seulement dans ton pays d'origine, mais aussi ailleurs. Ce genre d'amalgame est-il récurrent selon ton expérience, et l'as-tu rencontré ailleurs dans tes nombreuses installations, performances et résidences dans d'autres pays ? Une partie de ton succès à l'étranger au début n'est-elle pas due à un malentendu qui te voudrait l'béroïne de la résistance anti-communiste, sans tenir compte des contextes et des nuances, alors que tu as toujours combattu ces clichés?

S. I. : Les interprétations erronées de cette espèce sont encore relativement fréquentes, surtout quand elles proviennent d'un public mal informé. La France ne fait pas exception. Mais, même les experts « occidentaux » classent l'histoire de l'art de la Yougoslavie dans le cadre narratif de l'Europe de l'Est ; en conséquence, les pratiques critiques de l'art sont généralement perçues à travers la figure ou la position du dissident, c'est-à-dire de l'individu rebelle qui se bat pour les libertés fondamentales d'expression dans un système totalitaire. D’autre part, notre histoire commune se voit décomposée en une série de micro-histoires nationales. Ainsi, nos divers artistes, pratiques et phénomènes artistiques, sont renvoyés sous l'égide des « cultures nationales », ce qui va de pair avec la formation des États-nations. Au fait, les deux processus dérivent d'une acceptation consensuelle, souvent inconsciente et banalisée, d'un anticommunisme latent.

R. I. : Tu es une artiste sans galerie. Dans l'art contemporain qui est aussi commercial, il n'y a presque pas d'artistes sans galerie. Est-ce un choix de ta part, ou bien un concours de circonstances avec une longue histoire? Resterastu sans galeriste?

S. I. : Pour l'instant je résiste aux offres. Certes, j'ai un ami galeriste espagnol, qui est artiste lui-même et qui, occasionnellement, offre mes œuvres à des institutions publiques (musées). Je ne vends pas à des collectionneurs privés. J'ai commencé ma carrière d'artiste en Yougoslavie où il n'y avait ni galeries privées ni marché d'art développé. J'appartenais à une génération qui trouvait étrange l'idée de produire des œuvres d'art qui seraient vendues comme des produits de luxe. Nous étions conscients de l'importance sociale de notre travail et nous demandions un salaire comme tout le monde. Dalibor Martinis et moi l'avons bien exprimé dans un texte intitulé «Accord sur les conditions de la présentation publique du travail artistique » que nous avons rédigé ensemble en 1978 en tant que fondateurs du premier « Centre d'Art » 
Podroom. C'était une galerie gérée par douze artistes (parmi lesquels j'étais la seule femme-artiste!)

R. I. : Bien que tu avais déjà une belle notoriété et que tu avais fait le tour du monde avec ton travail, et faisais déjà partie de Documenta etc., ton exposition "Sweet violence » au MOMA à New York (décembre-mars 2012) t'a lancée dans la cour des grands. Depuis, les invitations importantes s'enchâेnent plus que jamais, le MUDAM à Luxembourg (juin-septembre 2012), le MAC/VAL à Ivry/Paris (octobre 2012) et bientôt Londres (décembre 20I2) t'invitent les uns après les autres pour d'importantes expositions. Est-ce que cette grande sollicitation change quelque chose dans ton engagement artistique pour le meilleur ou pour le pire, et si oui, quoi ? N'y a-t-il pas de danger d'usure?

S. I. : C'est une question que je me pose à moi-même tous les matins. Le problème du rapport entre la pratique de l'art critique (ou de n'importe quel art) et de son institutionnalisation n'est pas nouveau. Cela fait plus d'un siècle que les artistes appellent à l'abolition des musées et des salons qu'ils regardent comme des cimetières de l'art. Ils « descendent dans la rue » pour ne pas assister à leur propre enterrement. Les artistes conceptuels, quant à eux, cherchent à réduire la visibilité de leur art à des formes minimales allant jusqu'à sa dématérialisation totale, etc. D'autre part, les « institutions artistiques » (musées) continuent à accumuler des ouvres qui, à certains moments de l'histoire, avaient un potentiel critique et contestataire comme, par exemple, celles de Manet, Courbet, Delacroix, les premiers collages à thématique sociale ainsi que la Guernica de Picasso, etc. Je suis bien consciente du fait que la « muséification » a réduit la portée critique de mes ouvres mais j'espère qu'au

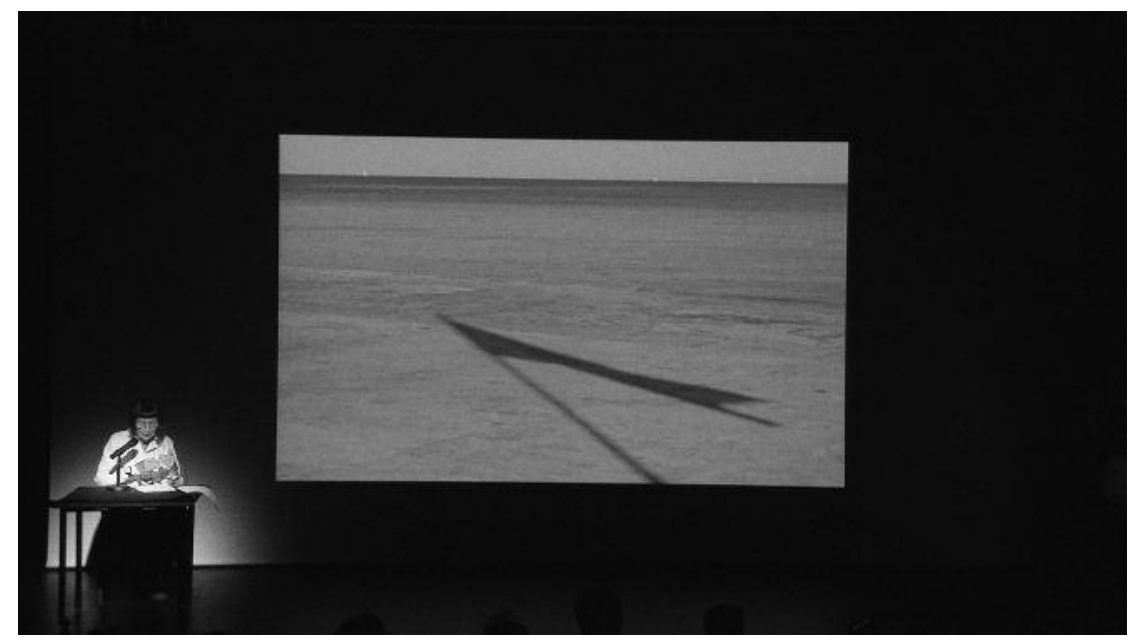

Rada Iveković dans la performance « Pourquoi un(e) artiste ne peut représenter un État-nation », MAC/VAL (C) Agnès Deschamps 
moment de leur création, elles avaient un potentiel militant. L'art ne peut jamais remplacer l'action politique mais il peut déclencher un débat public sur des sujets que le pouvoir ne souhaite pas problématiser. Mon projet « Lady Rosa of Luxembourg » en est un exemple. Dans le paradis capitaliste qu'est le Luxembourg, ce travail a suscité d'importantes controverses publiques et a même donné lieu à une action législative du parlement national.

R. I. : Revenons à tes débuts : tu avais fait l'académie des beaux arts à Zagreb. Enfant, tu dessinais bien - ce qui était d'ailleurs bien partagé dans la famille. L'un de nos grands-oncles, Oton Iveković (I 869-1939), avait été un peintre romantique en Croatie, et considéré comme "peintre national ». Alors que certains s'étaient orientés vers l'art contemporain parce qu'ils n'étaient pas très habiles à la peinture ou au dessin, ce n'était pas ton cas, tu étais "donée ». Tu as fait par la suite carrière chez un bon éditeur, et tu as formé le goût de générations en matière de couvertures et présentations de livres. Les tiennes étaient modernes, magnifiques, certaines sont restées légendaires et emblématiques d'une époque. Peux-tu rétrospectivement retracer l'bistoire esthétique et politique de ton revirement ? Pourquoi et comment en es-tu arrivée, avec tes amis, à te détourner des tableaux sur les murs, même excellents dans leur genre, car finalement vous avez été formés par de bons artistes que vous avez reniés et, à vos yeux, dépassés ? Quelle modernité représentiez-vous dans un pays (la Yougoslavie) où la peinture et la sculpture abstraite étaient soutenues par les autorités, ò̀ l'art était subventionné et les artistes ainsi que la culture en général aidés et souvent salariés?

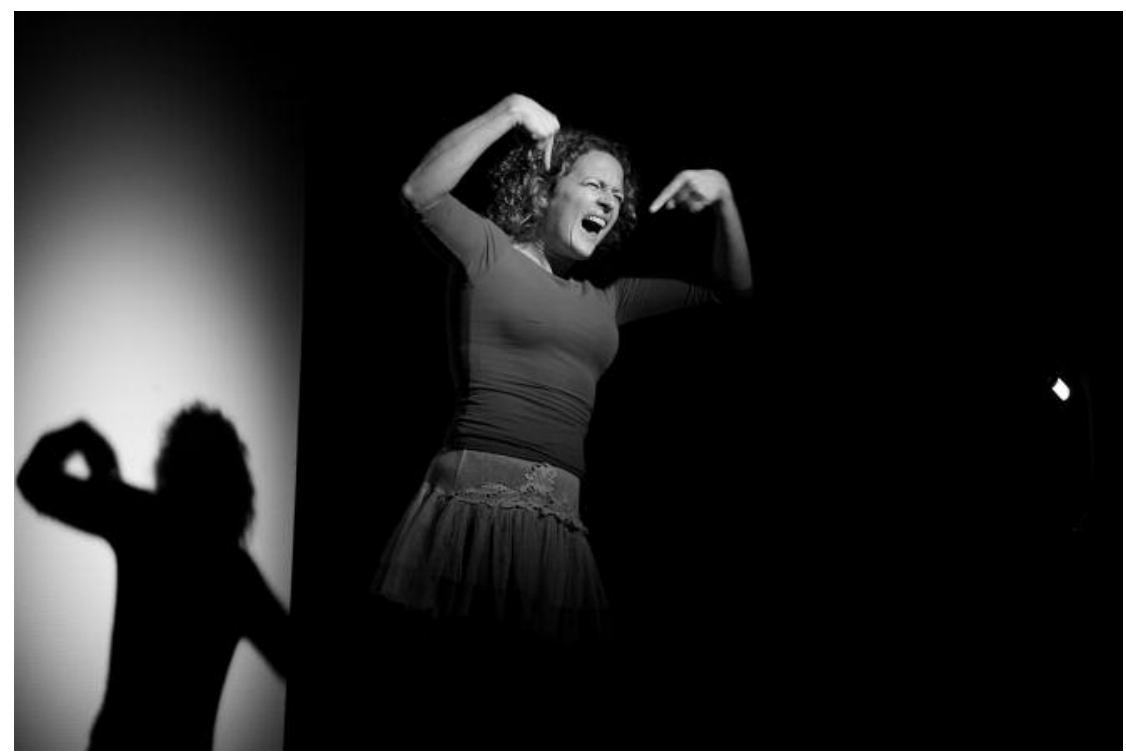

Isabelle Voizeux dans la performance «Pourquoi un(e) artiste ne peut représenter un État-nation » par Sanja Iveković, MAC/VAL (C Agnès Deschamps 
S. I. : La législation du socialisme autogestionnaire Yougoslave protégeait en principe l'artiste indépendant en tant que « travailleur culturel » dont le travail est utile à la société. Si l'artiste pouvait se prévaloir d'expositions individuelles ou collectives, il/elle pouvait présenter une demande d'octroi de statut (d'artiste indépendant) ce qui lui permettait de bénéficier d'une assurancemaladie et de faire valoir ses années d'ancienneté pour obtenir une retraite. En d'autres termes, nous n'avions pas à vendre nos œuvres d'art sur le marché pour prouver que nous étions des artistes «professionnels » comme c'est le cas aujourd'hui. Il suffisait être admis dans l'« Association des artistes plastiques de Croatie ». Je me souviens que mes amis artistes en Occident capitaliste étaient très étonnés qu'une telle chose puisse exister.

J'appartiens à la génération qui fit son apparition sur la scène (de l'art) au début des années 1970 et dont la démarche fut appelée « nouvelle pratique de l'art» (New art practice). Notre activité se déroulait la plupart du temps dans des galeries qui faisaient partie des centres culturels étudiants, mais parfois aussi dans des galeries publiques qui jouissaient d'un prestige considérable et qui souhaitaient présenter la scène d'avant-garde dans leurs programmes locaux et internationaux (par exemple le Musée d'Art Contemporain de Zagreb). S'il est vrai que d'un côté, notre pratique était marginalisée (les « centres culturels étudiants » étaient connus pour héberger des « pratiques d'art alternatives»), d'un autre côté, ce n'était pas des activités que l'on pourrait définir comme "dissidentes » étant donné qu'elles étaient - elles aussi, soutenues par des fonds publics. Les artistes non plus, qui y exerçaient leurs activités, ne se positionnaient pas comme des dissidents. Leurs efforts n'étaient pas dirigés contre un supposé « totalitarisme communiste noir », mais se voulaient critiques d'un gouvernement bureaucratique ancré sur le maintien à tout prix du statu quo. Donc, on pourrait dire, à juste titre, que ces militants de la scène contre-culturelle de l'époque prenaient le projet socialiste beaucoup plus au sérieux que l'élite politique officielle, imbue de cynisme. Les jeunes «travailleurs culturels » qui écrivaient pour la presse des jeunesses socialistes se voulaient les apôtres d'une « révolution permanente » et les artistes conceptuels demandaient dans certains de leurs manifestes « un art révolutionnaire pour une société révolutionnaire ». «La nouvelle pratique de l'art » était vraiment «nouvelle » dans le sens qu'elle posait, pour la première fois, des questions radicales sur la nature et la fonction de l'art lui-même, des questions sur l'« autonomie » dans un contexte " galerie-musée ", des questions touchant à l'influence de la logique de marché sur la production de l'œuvre d'art, etc. S'il est vrai que tout cela se trouvait également dans le programme des artistes occidentaux, l'idée même de la dématérialisation de l'œuvre d'art et, en général, d'un art qui abandonne les établissements officiels pour communiquer avec «le peuple» nous semble - elle - beaucoup plus proche de l'idée socialiste de la société. 


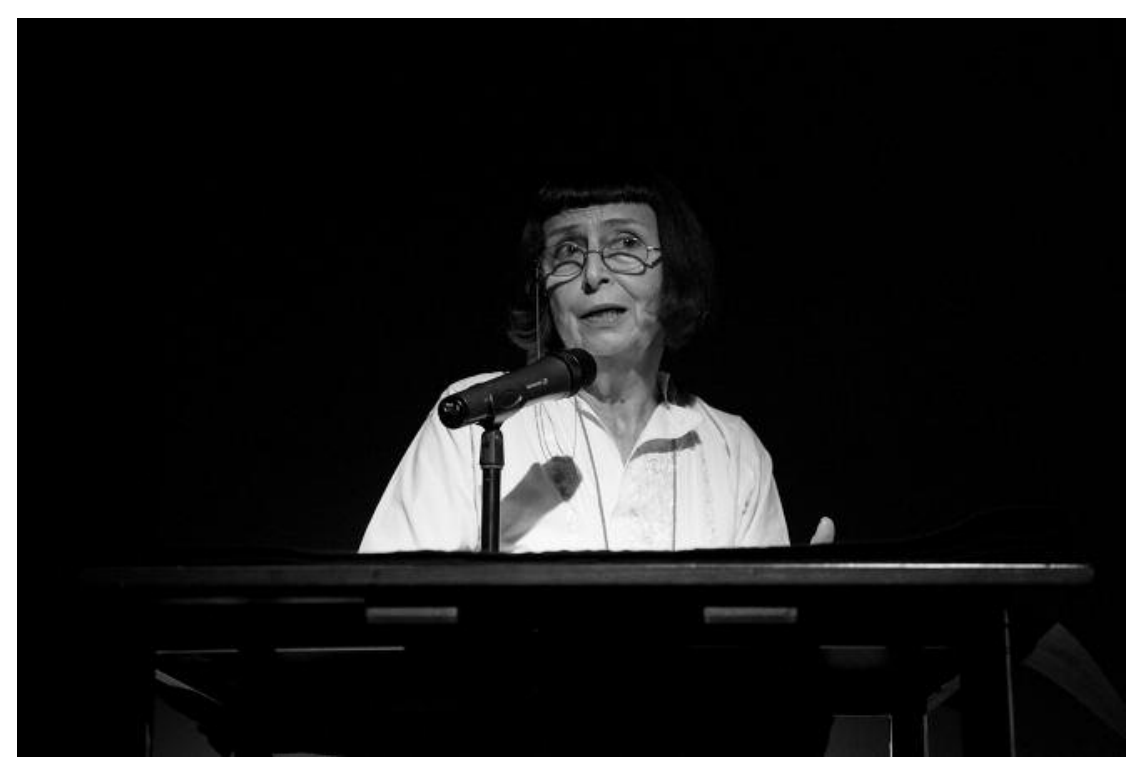

Rada Iveković dans la performance « Pourquoi un(e) artiste ne peut représenter un État-nation » par Sanja Iveković, MAC/VAL (C) Agnès Deschamps

R. I. : L'engagement féministe est intervenu à un certain moment dans ton travail. Depuis quand es-tu une artiste expressément féministe? Te souviens$t u d u$ contexte personnel ou politique de l'émergence de cette orientation? Quelles influences?

S. I. : Ma première rencontre avec l'art féministe date de la première exposition européenne d'art vidéo " 73 Trigon » qui s'est tenue à Graz, en Autriche. J'y ai participé et j'y ai vu les œuvres de Valie Export, Linda Benglis, Joan Jonas, Trisha Brown, Hermine Libéré. L’année suivante, j’ai pris part à Lausanne à la deuxième exposition internationale «Impact Art - Video Art » où j’ai pu voir les vidéos d'Eleanor Antin, de Martha Rosler, Yvonne Reiner et autres. Dans les revues d'art internationales que j'ai pu consulter - Flash Art, Artforum, Avalanche - le débat sur l'art féministe était déjà en cours. Tout cela m'inspirait beaucoup. Dans le cadre de ma deuxième exposition individuelle où j'ai présenté mes œuvres produites en 1975 j'ai donc voulu examiner ma propre condition de femme, mais également, le système dans lequel je vivais (y compris, en particulier, les aspects du système qui touchent à l'art). Donc, j'ai commencé par la pratique ; la théorie est venue plus tard. Ma formation féministe «théorique » a commencé dans les réunions de la section «Femme et société », fondée par un groupe de féministes (tu as été une des personnes clé du groupe !) et organisée dans le cadre de l'Association des sociologues de Croatie. Plus tard, en 1978, il y a eu la fameuse conférence féministe internationale Drug-ca Žena-Žensko pitanje- novi pristup ? 
(Camarade-femme : question féminine - nouvelle approche ?) qui s'est tenue au Centre culturel des étudiants (SKC) à Belgrade, organisée par la critique d'art Dunja Blažević et la sociologue Žarana Papić. Pour beaucoup de femmes qui y ont participé, l'événement a eu la signification du fameux " grand déclic ».

R. I. : Quel a été le rôle du soutien des femmes et des critiques d'art et curatrices dans ton travail ?

S. I. : Les groupes de femmes ont joué un rôle très important dans la construction de la scène civile en Yougoslavie car ils proposaient d'autres formes d'organisation et d'articulation d'intérêts par rapport aux courants (officiels) dominants, ou à la législation sociale. Je suis d'accord avec la thèse selon laquelle ce sont précisément les groupes de femmes qui ont le plus contribué au lancement des initiatives démocratiques. Les femmes ont été les premières à lancer des initiatives autonomes, en dehors des institutions officielles telles que l'Alliance socialiste, la Conférence pour l'activité sociale des femmes, la Ligue des Communistes, etc.

Pour moi, la coopération avec les organisations féministes a été une expérience inestimable. C'est suite à cette rencontre que j'ai commencé à changer et les contenus et les méthodes de mon travail artistique. Avant, je ne savais rien, par exemple, sur la violence envers les femmes car ce sujet n'était point présent dans le cercle d'artistes dans lequel j'évoluais. C'est pourquoi j'ai lancé en 1998 mon projet «La maison des femmes » (Ženska kuća) qui continue, au fait, comme «work in progress » jusqu'à ce jour, et qui évolue sous une variété

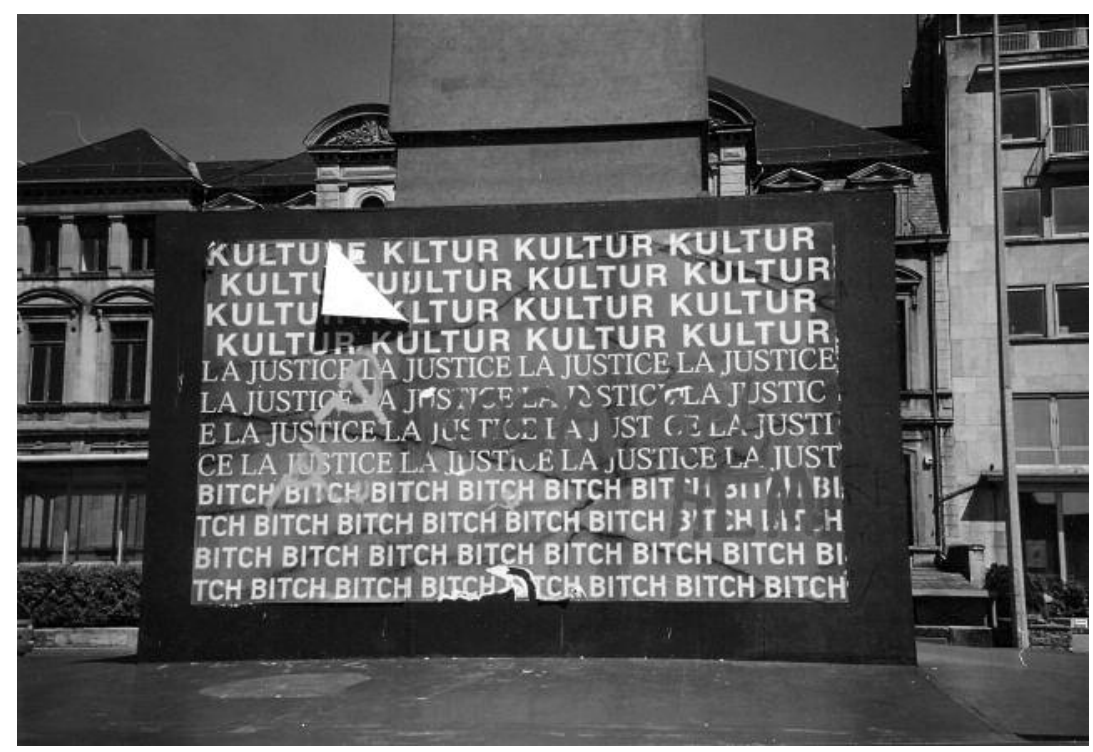

"Lady Rosa of Luxembourg » - Sanja Iveković, MUDAM, Luxembourg, 2001 
de formes et de formats. Il est mené en collaboration avec les femmes victimes de violence domestique, hébergées par l'unique institution dédiée à cette catégorie de personnes en Croatie - «La maison autonome des femmes» de Zagreb. Ce qui m'importait c'est que ces femmes ne soient pas seulement l'objet mais aussi les coréalisatrices du projet et de l'œuvre artistique. Ce travail n'aurait jamais pu se réaliser sans leur coopération dans les ateliers où elles créaient en les décalquant leurs propres masques et racontaient leurs histoires. Mon projet a également fait partie de la campagne de sensibilisation du public par ladite organisation féministe « Maison autonome des femmes » à la violence faite aux femmes. Cela se passait l'année où la Maison s'était trouvée à court de financement public et d'aide de l'État en faveur de ce refuge pour les femmes violentées. On peut signaler également que l'année du lancement du projet 1998 a coïncidé avec la proclamation par les Nations Unies du viol perpétré contre les membres de la population civile en contexte de guerre, comme crime de guerre international.

R. I. : Quels sont les artistes et orientations artistiques de par le monde qui t'intéressent actuellement? As-tu des modèles ou des figures que tu admires?

S. I. : Aujourd'hui, nous ne pouvons plus parler de courants artistiques, quoique les critiques d'art s'efforcent toujours de définir des tendances et de leur attribuer des noms tels que, par exemple « l'esthétique relationnelle » de Nicolas Bourrioud. Quant à moi, l'art qui m'intéresse est celui qui questionne et provoque et qui possède une dimension politique. Il y a un grand nombre de femmes et d'hommes-artistes, de la jeune génération surtout, dont le travail m'inspire et que j'apprécie beaucoup. Ce sont, pour la plupart, des artistes qui travaillent dans ou proviennent de pays que l'on situe encore à la " périphérie » (bien que les frontières entre «centre » et « périphérie » aient tendance à disparaitre). Pour ne citer que quelques noms, il s'agit de femmes-artistes telles que Tania Bruguera, Banu Cenetoglu, Milica Tomić, Philip Cesar, Yael Bartan, Jinos Taghizadeh, Rena Effendi... et parmi les artistes-hommes, de Rabih Mroué, Valide Ra'ad, Omer Fast, Jour Perjovshi... Je connais bien aussi les groupes «Chto delat ? » et le «Groupe Otholith » dont la contribution à la scène artistique contemporaine est extrêmement importante.

R. I. : Tes deux parents avaient été des résistants, partisans pendant la Deuxième guerre mondiale. Ils sont morts avant la disparition de la Yougoslavie. Ta mère Nera était une rescapée d'Auschrwitz; tu lui avais d'ailleurs consacré un grand projet à la Documenta de 2002 à Kassel, "Je cherche le numéro de ma mère ». As-tu été marquée par leurs histoires de guerre et de camps d'extermination? Ton option pour l'art conceptuel, que tu étais, avec ta génération, l'une des premières à introduire en Yougoslavie, est-elle en réaction à la génération de nos parents et de leurs valeurs politiques et esthétiques? 
S. I. : Je suis reconnaissante à mes parents (surtout à ma mère) d'avoir soutenu mes choix et mon travail artistique. Dans leur vie, ils ont fait preuve de beaucoup de courage et leur exemple m'a sans doute aidée à comprendre que dans la vie, il faut toujours se battre pour ses idées et ses idéaux. Je ne peux pas dire que mon choix de l'art conceptuel n'ait été qu'une réaction aux valeurs partagées par leur génération. Pendant les années 1960 et 1970, d'importants changements ont eu lieu dans le domaine de l'art, en Yougoslavie et dans le monde occidental. L'art conceptuel de cette période se caractérise par l'internationalisme ; il se manifeste aussi bien à New York qu’à Paris, à Prague, à Zagreb, à Düsseldorf... À l'époque de mes parents, c'est l'art abstrait qui faisait fonction d'art « officiel ». Nous sommes une génération qui a rompu avec la tradition moderniste.

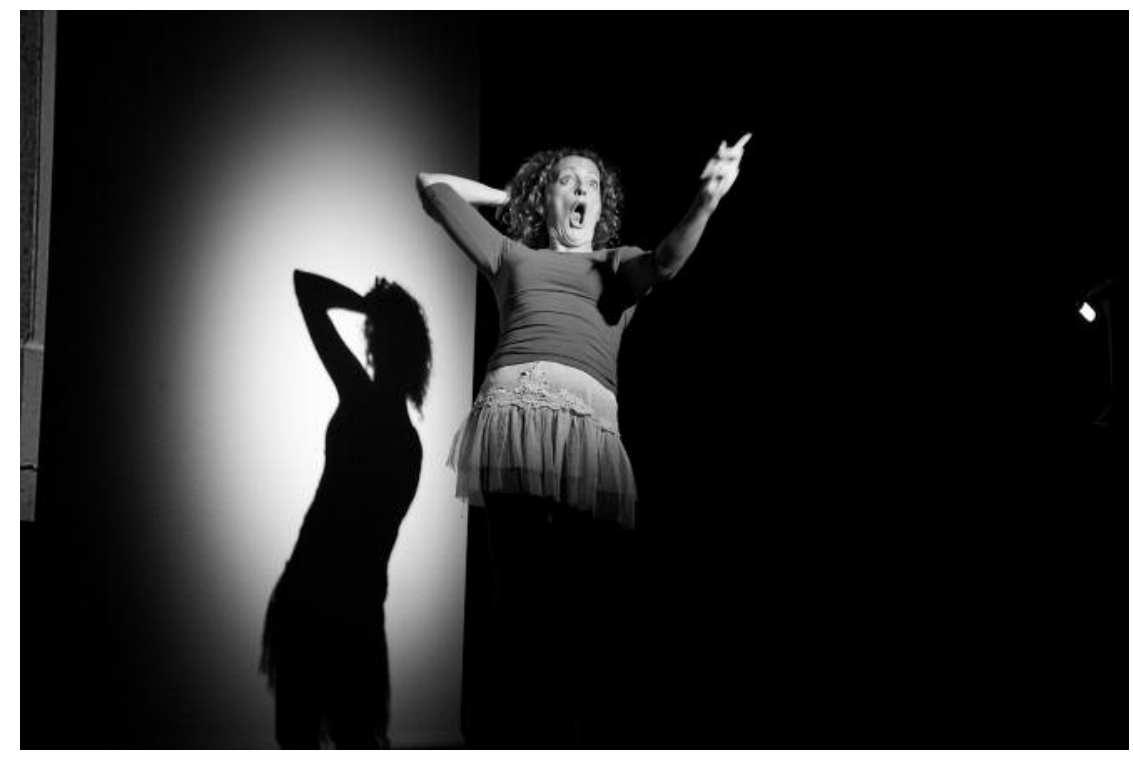

Isabelle Voizeux dans la performance "Pourquoi un(e) artiste ne peut représenter un État-nation » par Sanja Iveković, MAC/VAL @ Agnès Deschamps

R. I. : Tu as fait ta carrière d'artiste dans toute la Yougoslavie, et rapidement aussi à l'étranger. Tu faisais des installations à Belgrade et ailleurs dans le pays, tu étais soutenue, entres autres, par la critique d'art et curatrice dans une vision bien transnationale et cosmopolite avant la partition même $d u$ pays dans les années 1990. Peux-tu identifier une différence dans la manière que l'on a de traiter l'art, les artistes et la culture en Yougoslavie, en Croatie après son indépendance, et dans les divers pays au capitalisme débridé où tu as travaillé ? 

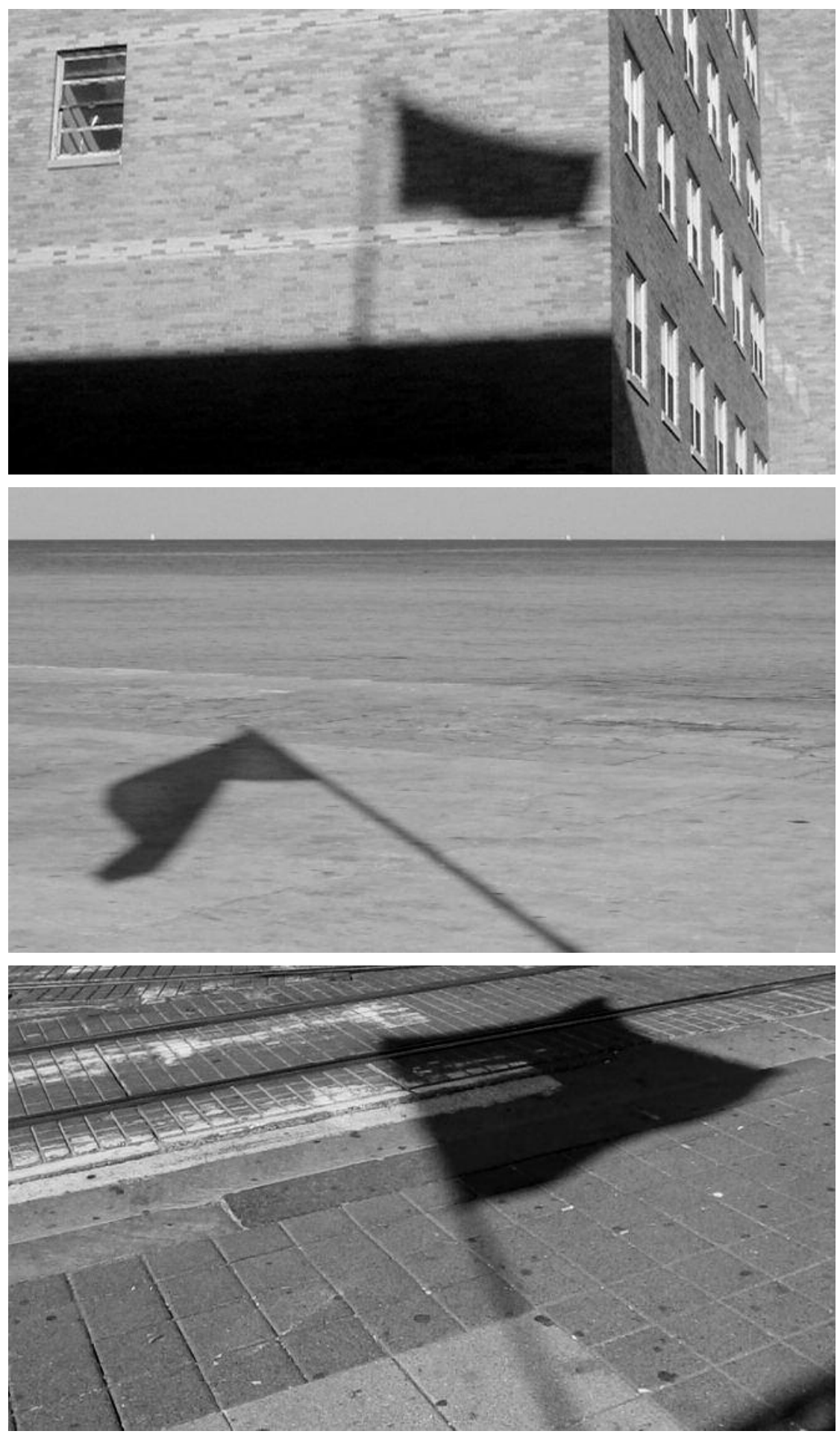

Images de la performance « Pourquoi un(e) artiste ne peut représenter un État-nation » par Sanja Iveković, MACNAL $\odot$ Agnès Deschamps 
S. I. : Mes œuvres prennent des formes très diverses parce que j'essaie toujours de trouver le moyen le plus efficace de faire passer le message dans un contexte donné. Je pense que l'état d'urgence qui caractérise notre époque, exige que les artistes soient extrêmement flexibles. Ces stratégies d'intervention (par opposition à un style individuel unique, à l'idée de l'artiste-génie couronné) sont précisément l'héritage féministe, tout à fait pertinent pour les pratiques artistiques contemporaines.

Nous assistons depuis quelque temps à un regain d'intérêt pour l'art conceptuel des années 1960 et 1970 en Europe de l'Est. Collectionneurs, curateurs indépendants et musées font preuve d'un intérêt particulier pour les premières pratiques féministes de femmes artistes originaires de cette région. Je me suis moi-même demandée si les pratiques conceptuelles des féministes de l'Europe de l'Est étaient en train de retourner à l'Ouest, en "rétrospective ", comme une pratique artistique marchandisée de plus, ou bien, était-ce par pur souci de "political correctness » que l'on présentait çà et là tel ou tel artiste d'Europe de l'Est dans les grandes expositions d'ensemble.

J'ai des sentiments mitigés au sujet de l'historisation de l'art féministe et je suis d'accord avec Linda Nochlin lorsqu'elle souligne la difficulté (je dirais presque l'impossibilité) de rendre sous forme de texte l'histoire de l'expérience de vie féministe et de la pratique de l'art féministe. Nochlin compare cela très pertinemment à la tentative de représenter un papillon en l'épinglant dans une vitrine. Je suis sceptique à ce sujet parce que je pense que la plupart des grandes expositions dans les musées entreprennent de «domestiquer » et de « pacifier » le potentiel critique de la pratique d'art féministe afin de le positionner sur le marché mondial de l'art. Ceci est, bien sûr, accompagné d'une tendance à idéaliser le passé, de sorte que malgré les formes nombreuses et souvent contradictoires qu'il épousait, l'art féministe dans ses débuts, est souvent dépeint comme un corps de travail homogène. Ceci est particulièrement vrai de la scène américaine.

Lorsqu'il s'agit de l'ex-Europe de l'Est on déplore que les organisateurs de ces expositions soient encore aujourd'hui, après quatre décennies, peu enclins à revoir cette pratique. Il en résulte qu'il n'y a toujours pas de bonne analyse de cette période. Les analyses écrites ou qui auraient pu l'être, par les protagonistes elles-mêmes, ne sont toujours pas disponibles dans les bibliographies «mainstream». Pour moi en tant que personne de cette région (bien que la Yougoslavie n'ait été incluse dans l'« Europe de l'Est » que rétrospectivement suite à une amnésie historique), il s'agit d'une question particulièrement douloureuse. Quand je me retrouve parmi le très petit nombre de femmes artistes de cette région, je me sens mal à l'aise, déçue et parfois en colère. 
Car si nous prenons comme exemple la plus grande exposition du genre jusqu'à ce jour «Wack! Art and the Feminist Revolution » (à laquelle j'ai été invitée à participer), on peut bien se poser la question si ce grand événement a été organisé dans le seul but de réaffirmer l'idée de domination de la pratique féministe états-unienne sur la scène internationale.

Cependant, il y a d'autres exemples. J'ai aussi participé à l'exposition espagnole "Gender Battle / A batalla dos xeneros » (cGac, Santiago de Compostela) dont le curateur a su montrer que le mouvement féministe a produit des ouvres splendides dans des milieux en marge du marché mondial de l'art, dans les pays d'Amérique du Sud, par exemple.

Retrouvez une vidéo de la performance de Sanja Iveković «Pourquoi un(e) artiste ne peut représenter un État-nation » sur le site de la revue, http://conflits.revues.org/18551 